\title{
Numerical Treatments For The Space- Time Fractional Fokker- Planck Equation By Means of Homotopy Perturbation Natural Transform Method
}

\author{
V.G.Gupta ${ }^{1}$ and Pramod Kumar ${ }^{2}$ \\ ${ }^{1,2}$ Department of Mathematics, University of Rajasthan, Jaipur-302024, India
}

\begin{abstract}
In the present paper, an efficient approach based on homotopy perturbation method by using natural transform is adopted to solve some linear and non-linear space-time fractional Fokker-Planck Equations (FPE) in closed form. The space and time fractional derivatives are considered in Caputo sense. The homotopy perturbation natural transform method is a combined form of natural transform, homotopy perturbation method and He's polynomials. The method gives an analytical solution in the form of a convergent series with easily computable components, requiring no linearization or small perturbation. The numerical result shows the effectiveness and good accuracy of the method.
\end{abstract}

Keywords: Fokker-Planck Equation, Caputo fractional derivative, Natural transform, Homotopy perturbation method.

\section{Introduction}

The Fokker-Planck equation (FPE) was introduced by Adriaan Fokker and Max Planck to describe the Brownian motion [5] of particles and the diffusion mode of chemical reactions [18], are now largely employed in various generalized forms, including solid-state Physics, Chemistry, circuit theory, engineering, and biology [24]. The Fokker-Planck equation arises in kinetic theory [8], where it describes the evolution of the one particle distribution function of a dilute gas with long range collisions, such as Coulomb gas. For the some applications of this type of equations one can refer the works of $\mathrm{He}$ and $\mathrm{Wu}$ [15], Jumarie [16], Xu et al [28].The fractional Fokker-Planck equation has recently been treated by a number of authors $[6,9,19,20,21]$.

The general FPE for the motion of a concentration field $u(x, t)$ of one space variable $x$ and the time thas the

form [24],

$$
\frac{\partial u(x, t)}{\partial t}=\left[-\frac{\partial}{\partial x} A(x)+\frac{\partial^{2}}{\partial x^{2}} B(x)\right] u(x, t)
$$

with initial conditions

$$
u(x, 0)=f(x) \quad, x \in R
$$

Where $A(x)$ and $B(x)>0$ are called the drift and diffusion coefficients. Mathematically, (1.1) is a linear second-order partial differential equation of parabolic type. The drift and diffusion coefficients may also depends on time as

$$
\frac{\partial u(x, t)}{\partial t}=\left[-\frac{\partial}{\partial x} A(x, t)+\frac{\partial^{2}}{\partial x^{2}} B(x, t)\right] u(x, t)
$$

There is a more general form of FPE which is called the nonlinear Fokker-Planck equation. The nonlinear FPE has important applications in various areas such as plasma physics, population dynamics, surface physics, engineering, nonlinear Hydrodynamics, pattern formation and so forth [7]. In one variable case, the nonlinear FPE can be written as $\frac{\partial u(x, t)}{\partial t}=\left[-\frac{\partial}{\partial x} A(x, t, u)+\frac{\partial^{2}}{\partial x^{2}} B(x, t, u)\right] u(x, t)$

with the initial condition

$$
u(x, 0)=f(x) \quad, x \in R
$$

Due to the vast range of applications of the Fokker-Planck equations, a lot of work has been done to find the numerical solutions of the equation. In this context, the works done by Buet. et. al. [3], Harrison [11], Vanjara [27], Yildirim [29], and Zarzano [30], are worth mentioning.

Our concern in this paper is to consider the numerical solution of the FPE with space and time- fractional derivatives of the form 


$$
\begin{aligned}
\frac{\partial^{\alpha} u(x, t)}{\partial t^{\alpha}} & =\left[-\frac{\partial^{\beta}}{\partial x^{\beta}} A(x, t, u)+\frac{\partial^{2 \beta}}{\partial x^{2 \beta}} B(x, t, u)\right] u(x, t) \\
t & >0, x>0 ; 0<\alpha, \beta \leq 1
\end{aligned}
$$

where $\alpha$ and $\beta$ are parameters describing the order of the fractional time and space derivatives, respectively. The function $u(x, t)$ is assumed to be a casual function of $\alpha=1$ and $\beta=1$, the fractional FPE (1.6) is reduced to the classical nonlinear FPE given by (1.4). The objective of present paper is to obtain closed form solution of linear and nonlinear space and time fractional FPE using homotopy perturbation natural transform method.

\section{Preliminaries}

For the concept of fractional derivatives, we will adopt Caputo's definition which is a modification of the Riemann-Liouville definition and has the advantage of dealing properly with initial value problems in which the initial conditions are given in terms of the field variable and their integral order which is the case in most physical processes. Some basic definitions and properties of fractional calculus theory which we have used in this paper are given in this section.

Definition 2.1 A real function $f(x), x>0$ is said to be in the space $C_{\mu}, \mu \in R$, if there exist a real number $p(>\mu)$ such that $\mathrm{f}(\mathrm{x})=\mathrm{x}^{\mathrm{p}} \mathrm{f}_{1}(\mathrm{x})$, where $\mathrm{f}_{1}(\mathrm{x}) \in \mathrm{C}[0, \infty)$, and it is said to be in the space $\mathrm{C}_{\mu}^{\mathrm{m}}$ iff $\mathrm{f}^{(\mathrm{m})} \in \mathrm{C}_{\mu}, \mathrm{m} \in \mathrm{N} \cup\{0\}$.

Definition 2.2.The Riemann-Liouville fractional integral operator of order $\alpha \geq 0$ of a function $f \in C_{\mu}, \mu \geq-1$ is defined as

$$
\begin{aligned}
\mathrm{J}^{\alpha} \mathrm{f}(\mathrm{x}) & =\frac{1}{\Gamma \alpha} \int_{0}^{\mathrm{x}}(\mathrm{x}-\mathrm{t})^{\alpha-1} \mathrm{f}(\mathrm{t}) \mathrm{dt}, \alpha>0, \mathrm{x}>0 \\
\mathrm{~J}^{0} \mathrm{f}(\mathrm{x}) & =\mathrm{f}(\mathrm{x})
\end{aligned}
$$

Properties of the operator $\mathbf{J}^{\alpha}$ can be found in [22,23,25], we mention only the following:

$$
\begin{aligned}
& \mathbf{J}^{\alpha} \mathbf{J}^{\beta} \mathrm{f}(\mathrm{x})=\mathrm{J}^{\alpha+\beta} \mathrm{f}(\mathrm{x}) \\
& \mathrm{J}^{\alpha} \mathbf{J}^{\beta} \mathrm{f}(\mathrm{x})=\mathbf{J}^{\beta} \mathrm{J}^{\alpha} \mathrm{f}(\mathrm{x}) \\
& \mathbf{J}^{\alpha} \mathrm{x}^{\gamma}=\frac{\Gamma(\gamma+1)}{\Gamma(\alpha+\gamma+1)} \mathrm{x}^{\alpha+\gamma}
\end{aligned}
$$

For $\mathrm{f} \in \mathrm{C}_{\mu}, \mu \geq-1, \alpha, \beta \geq 0$ and $\gamma>-1$.

Definition 2.3.The fractional derivative of $f(x)$ in the Caputo sense is defined as [4]

$$
D_{*}^{\alpha} f(x)=J^{m-\alpha} D_{*}^{m} f(x)=\frac{1}{\Gamma(m-\alpha)} \int_{0}^{x}(x-t)^{m-\alpha-1} f^{(m)}(t) d t
$$

For $\mathrm{m}-1<\alpha \leq \mathrm{m}, \mathrm{m} \in \mathrm{N}, \mathrm{x}>0, \mathrm{f} \in \mathrm{C}_{-1}^{\mathrm{m}}$.

Also, we need here three basic properties

$$
D_{*}^{\alpha} J^{\alpha} f(x)=f(x)
$$

$$
J^{\alpha} D_{*}^{\alpha} f(x)=f(x)-\sum_{k=0}^{m-1} f^{(k)}\left(0^{+}\right) \frac{x^{k}}{k !}, \quad x>0
$$


(iii)

$$
\mathrm{D}_{*}^{\alpha} \mathrm{x}^{\gamma}=\frac{\Gamma(\gamma+1)}{\Gamma(\gamma-\alpha+1)} \mathrm{x}^{\gamma-\alpha} ; \mathrm{x}>0, \gamma>0 .
$$

For $\mathrm{m}-1<\alpha \leq \mathrm{m}, \mathrm{m} \in \mathrm{N}, \mu \geq-1$ and $\mathrm{f} \in \mathrm{C}_{\mu}^{\mathrm{m}}$.

$$
t \geq 0
$$

Definition 2.4 The Natural Transform $R(s, u)$ of the function $f(t)$ for all $\quad$ is given by $[2,17]$

$$
N^{+}[f(t)]=R(s, u)=\frac{1}{u} \int_{0}^{\infty} e^{-s t} f(u t) d t \quad, s>0, u>0
$$

provided that integral on the right exist.

where $t$ and $u$ are time variable and $s$ is the frequency variable, provided the function $f(t)$ is defined in the set $A$ by

$$
A=\left\{f(t): \exists M, \tau_{1}, \tau_{2}>0,|f(t)|<M e^{1 / \tau_{j}} ; t \in(-1)^{j} \times[0, \infty)\right\}
$$

Where $M$ is a constant of finite number $\tau_{l}$ and $\tau_{2}$ may be finite or infinite. The discrete form of natural Transform is given by [2]

$$
N^{+}[f(t)]=R(s, u)=\sum_{n=0}^{\infty} \frac{n ! a_{n} u^{n}}{s^{n+1}}
$$

The inverse natural transformation is defined by [2] and [26]

$$
N^{-}[R(s, u)]=f(t)=\frac{1}{2 \pi i} \int_{c-i \infty}^{c-i \infty} e^{s t / u} R(s, u) d s
$$

The duality relation between Natural-Laplace transform and Natural- Sumudu transform is given by

$$
R(s, u)=\frac{1}{u} F\left(\frac{s}{u}\right) ; R(s, u)=\frac{1}{s} G\left(\frac{u}{s}\right)
$$

where $F(s)$ denotes the Laplace transform and $G(u)$ is the Sumudu transform. Properties of Natural transform can be seen in $[2,17,26]$, some are given below.

(i) If $R(s, u)$ is the Natural Transform of $f(t)$, then the natural transform of $f^{(n)}(t)$ is given by

$$
N^{+}\left[f^{(n)}(t)\right]=R_{n}(s, u)=\frac{s^{n}}{u^{n}} R(s, u)-\sum_{k=0}^{n} \frac{s^{n-k-1}}{u^{n-k}} f^{(k)}(0)
$$

(ii) When $f(t)=\delta(t)$, the Dirac delta function, the natural transform becomes

$$
\begin{gathered}
N^{+}[\delta(t)]=R(s, u)=\frac{1}{u} \\
\text { and when } \begin{array}{c}
f(t)=\frac{t^{n-1}}{\Gamma n} ; n>0 \text {, the natural transform is } \\
N^{+}\left[\frac{t^{n-1}}{\Gamma n}\right]=\frac{u^{n-1}}{s^{n}}
\end{array}
\end{gathered}
$$

(iii). If $R(s, u)$ is the natural transform of the function $f(t)$ then the natural transform of fractional integral of order $\beta$ is defined by

$$
N^{+}\left[D^{-\beta} f(t)\right]=\frac{u^{\beta}}{s^{\beta}} R(s, u)
$$

(iv). If $R(s, u)$ is the natural transform of the function $f(t)$ then the natural transform of fractional derivative of order $\beta$ is defined by 


$$
N^{+}\left[D^{\beta} f(t)\right]=\frac{s^{\beta}}{u^{\beta}} R(s, u)-\sum_{k=0}^{n} \frac{s^{n-k-1}}{u^{n-k}} f^{(k)}(0), n-1 \leq \beta<1
$$

\section{Solution of Fractional Differential Equations by Homotopy Perturbation Natural Transform Method.}

The homotopy perturbation method [HPM], introduced by He [12-15], is a series expansion method used in the solution of nonlinear partial differential equations. The HPM uses a so-called convergence-control parameter to guarantee the convergence of approximation series over a given interval of physical parameters.

To illustrate the basic idea of this method, we consider a general fractional nonlinear non-homogenous partial differential equation with initial conditions of the form

$$
\begin{array}{ll}
D_{t}^{\alpha} u(x, t)=R u(x, t)+N u(x, t)+g(x, t), & \alpha>0 \\
D_{0}^{\alpha} u(x, 0)=f_{k} & ,[k=0,1, \ldots, n-1] \\
D_{0}^{n} u(x, 0)=0 & , n=[\alpha]
\end{array}
$$

Where $g(x, t)$ is the source term, $N$ represents the general nonlinear differential operator and $R$ is the linear differential operator, $D_{t}^{\alpha} u(x, t)$ is the Caputo's fractional derivative of the function $u(x, t)$.

Applying natural transform of both sides of (3.1), we get

$$
\begin{aligned}
& N^{+}\left[D_{t}^{\alpha} u(x, t)\right]=N^{+}[R u(x, t)+N u(x, t)+g(x, t)] \\
& N^{+}[u(x, t)]=\frac{u^{\alpha}}{s^{\alpha}} N^{+}[R u(x, t)+N u(x, t)+g(x, t)]+\frac{u^{\alpha}}{s^{\alpha}} \sum_{k=0}^{n} \frac{s^{n-k-1}}{u^{n-k}} f^{(k)}(0)
\end{aligned}
$$

Operating with the inverse natural transform on both sides of (3.4), we get

$$
u(x, t)=G(x, t)+N^{-}\left[\frac{u^{\alpha}}{s^{\alpha}} N^{+}\{R u(x, t)+N u(x, t)\}\right]
$$

Where $G(x, t)$ represents the term arising from the source term and the prescribed initial condition.

Now we apply the homotopy perturbation method, the basic assumption is that the solutions can be written as a power series in $p$

$$
u(x, t)=\sum_{n=0}^{\infty} p^{n} u_{n}(x, t)=u_{0}+p u_{1}+p^{2} u_{2}+p^{3} u_{3}+\ldots
$$

and the nonlinear term can be decomposed as

$$
N u(x, t)=\sum_{n=0}^{\infty} p^{n} H_{n}(u)
$$

where $p \in[0,1]$ is an embedding parameter. $H_{n}(u)$ is He's polynomials [10] which is given by

$$
H_{n}\left(u_{0}, \ldots, u_{n}\right)=\frac{1}{n !} \frac{\partial^{n}}{\partial p^{n}}\left[N\left(\sum_{i=0}^{\infty} p^{i} u_{i}\right)\right]_{p=0}
$$

Substituting (3.6) and (3.7) in (3.5), we get

$$
\sum_{n=0}^{\infty} p^{n} u_{n}(x, t)=G(x, t)+p\left[N^{-}\left\{\frac{u^{\alpha}}{s^{\alpha}} N^{+}\left(R \sum_{n=0}^{\infty} p^{n} u_{n}(x, t)+\sum_{n=0}^{\infty} p^{n} H_{n}(u)\right)\right\}\right]
$$

which shows the coupling of the natural transform and the HPM by using He's polynomials.

Equating the terms with identical powers in $p$, we obtain the following components 


$$
\begin{array}{ll}
p^{0}: & u_{0}(x, t)=G(x, t) \\
p^{1}: & u_{1}(x, t)=N^{-}\left[\frac{u^{\alpha}}{s^{\alpha}} N^{+}\left\{R u_{0}(x, t)+H_{0}(u)\right\}\right] \\
p^{2}: & u_{2}(x, t)=N^{-}\left[\frac{u^{\alpha}}{s^{\alpha}} N^{+}\left\{R u_{1}(x, t)+H_{1}(u)\right\}\right]
\end{array}
$$

and similarly

$$
p^{n}: u_{n}(x, t)=N^{-}\left[\frac{u^{\alpha}}{s^{\alpha}} N^{+}\left\{R u_{n-1}(x, t)+H_{n-1}(u)\right\}\right]
$$

Finally, we approximate the analytical solution $u(x, t)$ by truncated series

$$
u(x, t)=\lim _{N \rightarrow \infty} \sum_{n=0}^{N} u_{n}(x, t)
$$

However we have seen in many cases, the exact solution in a closed form may be obtained. This method does not resort to linearization or assumption of weak nonlinearity, the solution generalized in the form of general solution and it is more realistic compare to the other methods of simplify the physical problems, also the decomposition series solutions generally converges very rapidly. Abbaoui and Cherruault [1], has defined the classical approach of convergence of this type of series.

\section{IV.Solution of Fokker-Plank Fractional Differential Equations}

Solution of following nonlinear space-time fractional Fokker-Planck equation (FPE)

$$
\begin{aligned}
& \frac{\partial^{\alpha} u(x, t)}{\partial t^{\alpha}}=\left[-\frac{\partial^{\beta}}{\partial x^{\beta}} A(x, t, u)+\frac{\partial^{2 \beta}}{\partial x^{2 \beta}} B(x, t, u)\right] u(x, t) \\
& t>0, x>0,0<\alpha, \beta \leq 1
\end{aligned}
$$

with the initial conditions

$$
u(x, 0)=f(x)
$$

Applying the natural transform of both sides of (4.1), subject to initial conditions (4.2), we have

$$
N^{+}[u(x, t)]=\frac{1}{s} f(x)+\frac{u^{\alpha}}{s^{\alpha}} N^{+}\left[-\frac{\partial^{\beta}}{\partial x^{\beta}} A(x, t, u) u(x, t)+\frac{\partial^{2 \beta}}{\partial x^{2 \beta}} B(x, t, u) u(x, t)\right]
$$

taking the inverse natural transform

$$
u(x, t)=f(x)+N^{-}\left[\frac{u^{\alpha}}{s^{\alpha}} N^{+}\left\{-\frac{\partial^{\beta}}{\partial x^{\beta}} \sum_{n=0}^{\infty} A_{n}+\frac{\partial^{2 \beta}}{\partial x^{2 \beta}} \sum_{n=0}^{\infty} B_{n}\right\}\right]
$$

where

$$
\begin{aligned}
& \sum_{n=0}^{\infty} A_{n}=A(x, t, u) \cdot u(x, t) \\
& \sum_{n=0}^{\infty} B_{n}=B(x, t, u) \cdot u(x, t)
\end{aligned}
$$

Using Homotopy Perturbation method, we get

$$
\sum_{n=0}^{\infty} p^{n} u_{n}(x, t)=f(x)+p\left[N^{-}\left\{\frac{u^{\alpha}}{s^{\alpha}} N^{+}\left(-\sum_{n=0}^{\infty} p^{n} H_{n}(x, t, u)+\sum_{n=0}^{\infty} p^{n} H_{n}^{1}(x, t, u)\right)\right\}\right]
$$


Where $H_{n}(x, t, u)$ and $H_{n}^{1}(x, t, u)$ are He's polynomial that represents the nonlinear term. The He's polynomial are given by

$$
\begin{aligned}
& \sum_{n=0}^{\infty} H_{n}(x, t, u)=\frac{\partial^{\beta}}{\partial x^{\beta}}\left(\sum_{n=0}^{\infty} A_{n}\right) \\
& \sum_{n=0}^{\infty} H_{n}^{1}(x, t, u)=\frac{\partial^{2 \beta}}{\partial x^{2 \beta}}\left(\sum_{n=0}^{\infty} B_{n}\right)
\end{aligned}
$$

Using the (4.6), we can collect the coefficients of different powers of $p$

$$
\begin{aligned}
& p^{0}: u_{0}(x, t)=f(x) \\
& p^{1}: u_{1}(x, t)=N^{-}\left[\frac{u^{\alpha}}{s^{\alpha}} N^{+}\left\{-H_{0}+H_{0}^{1}\right\}\right] \\
& p^{2}: u_{2}(x, t)=N^{-}\left[\frac{u^{\alpha}}{s^{\alpha}} N^{+}\left\{-H_{1}+H_{1}^{1}\right\}\right]
\end{aligned}
$$

and similarly

$$
p^{n}: u_{n}(x, t)=N^{-}\left[\frac{u^{\alpha}}{s^{\alpha}} N^{+}\left\{-H_{n-1}+H_{n-1}^{1}\right\}\right]
$$

Finally, we approximate the analytical solution $u_{n}(x, t)$ by truncated series [1]

$$
u(x, t)=\lim _{N \rightarrow \infty} \sum_{n=0}^{N} u_{n}(x, t)
$$

\section{Numerical Examples}

In this section, we will apply the method to solve linear and nonlinear fractional Fokker-Planck equation by the Homotopy perturbation natural transform method [HPNTM].

Example1. Consider the following space-time fractional nonlinear Fokker-Planck equation

$$
D_{t}^{\alpha} u(x, t)=-D_{x}^{\beta}\left(\frac{4 u^{2}}{x}-\frac{x u}{3}\right)+D_{x}^{2 \beta} u^{2}, 0<\alpha, \beta \leq 1
$$

subject to initial condition

$$
u(x, 0)=x^{2}
$$

Taking the natural transform of both sides, we get

$$
\begin{aligned}
& {\left[\frac{s^{\alpha}}{u^{\alpha}} N^{+}(u(x, t))-x^{2} \frac{s^{\alpha-1}}{u^{\alpha}}\right]=N^{+}\left[-D_{x}^{\beta}\left(\frac{4 u^{2}}{x}-\frac{x u}{3}\right)+D_{x}^{2 \beta} u^{2}\right]} \\
& N^{+}(u(x, t))=\frac{x^{2}}{s}+\frac{u^{\alpha}}{s^{\alpha}} N^{+}\left[-D_{x}^{\beta}\left(\frac{4 u^{2}}{x}-\frac{x u}{3}\right)+D_{x}^{2 \beta} u^{2}\right]
\end{aligned}
$$

Operating the inverse natural transform of both sides, we get

$$
u(x, t)=x^{2}+N^{-}\left[\frac{u^{\alpha}}{s^{\alpha}} N^{+}\left[-D_{x}^{\beta}\left(\frac{4 u^{2}}{x}-\frac{x u}{3}\right)+D_{x}^{2 \beta} u^{2}\right]\right]
$$

Then applying the homotopy perturbation method, we get 


$$
\sum_{n=0}^{\infty} p^{n} u_{n}=x^{2}+p\left[N^{-}\left[\frac{u^{\alpha}}{s^{\alpha}} N^{+}\left[-D_{x}^{\beta}\left(\frac{4 \sum_{n=0}^{\infty} p^{n} H_{n}(u)}{x}-\frac{x \sum_{n=0}^{\infty} p^{n} u_{n}}{3}\right)+D_{x}^{2 \beta} \sum_{n=0}^{\infty} p^{n} H_{n}(u)\right]\right]\right]
$$

On using the method defined in section IV, we get the coefficients of $p$

$$
\begin{aligned}
& p^{0}: u_{0}(x, t)=x^{2} \\
& p^{1}: u_{1}(x, t)=\left(\frac{24}{\Gamma(5-2 \beta)} x^{4-2 \beta}-\frac{22}{\Gamma(4-\beta)} x^{3-\beta}\right) \frac{t^{\alpha}}{\Gamma(\alpha+1)} \\
& p^{2}: u_{2}(x, t)=\left(\begin{array}{l}
\frac{506 \Gamma(5-\beta)}{3 \Gamma(5-2 \beta) \Gamma(4-\beta)} x^{4-2 \beta}-\frac{184 \Gamma(6-2 \beta)}{\Gamma(6-3 \beta) \Gamma(5-2 \beta)} x^{5-3 \beta} \\
-\frac{44 \Gamma(6-\beta)}{\Gamma(6-3 \beta) \Gamma(4-\beta)} x^{5-3 \beta}+\frac{48 \Gamma(7-2 \beta)}{\Gamma(7-4 \beta) \Gamma(5-2 \beta)} x^{6-4 \beta}
\end{array}\right) \frac{t^{2 \alpha}}{\Gamma(1+2 \alpha)}
\end{aligned}
$$

Hence

$$
\begin{gathered}
u(x, t)=u_{0}(x, t)+u_{1}(x, t)+u_{2}(x, t)+\ldots \\
=x^{2}+\left(\frac{24}{\Gamma(5-2 \beta)} x^{4-2 \beta}-\frac{22}{\Gamma(4-\beta)} x^{3-\beta}\right) \frac{t^{\alpha}}{\Gamma(\alpha+1)}+\left[\frac{506 \Gamma(5-\beta)}{3 \Gamma(5-2 \beta) \Gamma(4-\beta)} x^{4-2 \beta}\right. \\
\left.\quad-\frac{184 \Gamma(6-2 \beta)}{\Gamma(6-3 \beta) \Gamma(5-2 \beta)} x^{5-3 \beta}-\frac{44 \Gamma(6-\beta)}{\Gamma(6-3 \beta) \Gamma(4-\beta)} x^{5-3 \beta}+\frac{48 \Gamma(7-2 \beta)}{\Gamma(7-4 \beta) \Gamma(5-2 \beta)} x^{6-4 \beta}\right] \frac{t^{2 \alpha}}{\Gamma(1+2 \alpha)}+\ldots
\end{gathered}
$$

Remark 1: Setting $\alpha=\beta=1$ in (5.1), the equation reduces to classical nonlinear Fokker-Planck equation and the solution of the problem is as follows

$$
u(x, t)=x^{2}\left(1+t+\frac{t^{2}}{2 !}+\ldots\right)
$$

This solution is equivalent to the exact solution in a closed form

$$
u(x, t)=x^{2} e^{t}
$$

Table1.The results for exact solution (5.10) and the approximate solution (5.8) for (5.1) obtained using the homotopy perturbation natural transform method are shown in Table for $\alpha=\beta=1$

\begin{tabular}{|l|l|l|l|}
\hline $\mathrm{t}$ & $\mathrm{X}$ & HPNTM & EXACT \\
\hline 0.25 & 0.25 & 0.08025106 & 0.08025159 \\
\cline { 2 - 4 } & 0.50 & 0.32100420 & 0.32100640 \\
\cline { 2 - 4 } & 0.75 & 0.72225950 & 0.72246430 \\
\cline { 2 - 4 } & 1.00 & 1.28401700 & 1.28402500 \\
\hline 0.50 & 0.25 & 0.10302730 & 0.10304510 \\
\cline { 2 - 4 } & 0.50 & 0.41210940 & 0.41218030 \\
\cline { 2 - 4 } & 0.75 & 0.92724610 & 0.92740570 \\
\cline { 2 - 4 } & 1.00 & 1.64843800 & 1.64872100 \\
\hline 0.75 & 0.25 & 0.13217160 & 0.13231250 \\
\cline { 2 - 4 } & 0.50 & 0.52868650 & 0.52925000 \\
\cline { 2 - 4 } & 0.75 & 0.18954500 & 1.19081300 \\
\cline { 2 - 4 } & 1.00 & 2.11474600 & 2.11700000 \\
\hline
\end{tabular}

Example2: Consider the linear space- and time-fractional Fokker-Planck equation 
Subject to the initial condition

$$
D_{t}^{\alpha} u(x, t)=\left[-D_{x}^{\beta}(x)+D_{x}^{2 \beta}\left(\frac{x^{2}}{2}\right)\right] u(x, t), t>0, x>0 ; \quad 0<\alpha, \beta \leq 1
$$

$$
u(x, 0)=x
$$

By using the method defined in section-IV and illustrated in Example 5.1, we get the coefficient of $p$

$$
\begin{aligned}
& p^{0}: u_{0}(x, t)=x \\
& p^{1}: u_{1}(x, t)=\left(\frac{3}{\Gamma(4-2 \beta)} x^{3-2 \beta}-\frac{2}{\Gamma(3-\beta)} x^{2-\beta}\right) \frac{t^{\alpha}}{\Gamma(\alpha+1)} \\
& p^{2}: u_{2}(x, t)=\left(\begin{array}{l}
\frac{2 \Gamma(4-\beta)}{\Gamma(3-\beta) \Gamma(4-2 \beta)} x^{3-2 \beta}-\frac{3 \Gamma(5-2 \beta)}{\Gamma(4-2 \beta) \Gamma(5-3 \beta)} x^{4-3 \beta} \\
-\frac{\Gamma(5-\beta)}{\Gamma(3-\beta) \Gamma(5-3 \beta)} x^{4-3 \beta}+\frac{3 \Gamma(6-2 \beta)}{2 \Gamma(4-2 \beta) \Gamma(6-4 \beta)} x^{5-4 \beta}
\end{array}\right) \frac{t^{2 \alpha}}{\Gamma(1+2 \alpha)} \\
& p^{3}: u_{3}(x, t)=\left(\begin{array}{l}
-\frac{2 \Gamma(4-\beta) \Gamma(5-2 \beta)}{\Gamma(3-\beta) \Gamma(4-2 \beta) \Gamma(5-3 \beta)} x^{4-3 \beta}+\frac{3 \Gamma(5-2 \beta) \Gamma(6-3 \beta)}{\Gamma(4-2 \beta) \Gamma(5-3 \beta) \Gamma(6-4 \beta)} x^{5-4 \beta} \\
+\frac{\Gamma 5-\beta \Gamma(6-3 \beta)}{\Gamma(3-\beta) \Gamma(5-3 \beta) \Gamma(6-4 \beta)} x^{5-4 \beta}+\frac{\Gamma(4-\beta) \Gamma(6-2 \beta)}{\Gamma(3-\beta) \Gamma(4-2 \beta) \Gamma(6-4 \beta)} x^{5-4 \beta} \\
-\frac{3 \Gamma(6-2 \beta) \Gamma(7-4 \beta)}{2 \Gamma(4-2 \beta) \Gamma(6-4 \beta) \Gamma(7-5 \beta)} x^{6-5 \beta}-\frac{\Gamma(5-\beta) \Gamma(7-3 \beta)}{2 \Gamma(3-\beta) \Gamma(5-3 \beta) \Gamma(7-5 \beta)} x^{6-5 \beta} \\
-\frac{3 \Gamma(5-2 \beta) \Gamma(7-3 \beta)}{2 \Gamma(4-2 \beta) \Gamma(5-3 \beta) \Gamma(7-5 \beta)} x^{6-5 \beta}+\frac{3 \Gamma(6-2 \beta) \Gamma(8-4 \beta)}{4 \Gamma(4-2 \beta) \Gamma(6-4 \beta) \Gamma(8-6 \beta)} x^{7-6 \beta}
\end{array}\right) \quad \frac{t^{3 \alpha}}{\Gamma(1+3 \alpha)}
\end{aligned}
$$

Hence

$$
\begin{aligned}
u(x, t)=x+ & \left(\frac{3}{\Gamma(4-2 \beta)} x^{3-2 \beta}-\frac{2}{\Gamma(3-\beta)} x^{2-\beta}\right) \frac{t^{\alpha}}{\Gamma(\alpha+1)}+ \\
& \left(\begin{array}{l}
\frac{2 \Gamma(4-\beta)}{\Gamma(3-\beta) \Gamma(4-2 \beta)} x^{3-2 \beta}-\frac{3 \Gamma(5-2 \beta)}{\Gamma(4-2 \beta) \Gamma(5-3 \beta)} x^{4-3 \beta} \\
-\frac{\Gamma(5-\beta)}{\Gamma(3-\beta) \Gamma(5-3 \beta)} x^{4-3 \beta}+\frac{3 \Gamma(6-2 \beta)}{2 \Gamma(4-2 \beta) \Gamma(6-4 \beta)} x^{5-4 \beta}
\end{array}\right) \frac{t^{2 \alpha}}{\Gamma(1+2 \alpha)}+ \\
& \left(\begin{array}{l}
-\frac{2 \Gamma(4-\beta) \Gamma(5-2 \beta)}{\Gamma(3-\beta) \Gamma(4-2 \beta) \Gamma(5-3 \beta)} x^{4-3 \beta}+\frac{3 \Gamma(5-2 \beta) \Gamma(6-3 \beta)}{\Gamma(4-2 \beta) \Gamma(5-3 \beta) \Gamma(6-4 \beta)} x^{5-4 \beta} \\
+\frac{\Gamma(5-\beta) \Gamma(6-3 \beta)}{\Gamma(3-\beta) \Gamma(5-3 \beta) \Gamma(6-4 \beta)} x^{5-4 \beta}+\frac{\Gamma(4-\beta) \Gamma(6-2 \beta)}{\Gamma(3-\beta) \Gamma(4-2 \beta) \Gamma(6-4 \beta)} x^{5-4 \beta} \\
-\frac{3 \Gamma(6-2 \beta) \Gamma(7-4 \beta)}{2 \Gamma(4-2 \beta) \Gamma(6-4 \beta) \Gamma(7-5 \beta)} x^{6-5 \beta}-\frac{\Gamma(5-\beta) \Gamma(7-3 \beta)}{2 \Gamma(3-\beta) \Gamma(5-3 \beta) \Gamma(7-5 \beta)} x^{6-5 \beta} \\
-\frac{3 \Gamma(5-2 \beta) \Gamma(7-3 \beta)}{2 \Gamma(4-2 \beta) \Gamma(5-3 \beta) \Gamma(7-5 \beta)} x^{6-5 \beta}+\frac{3 \Gamma(6-2 \beta) \Gamma(8-4 \beta)}{4 \Gamma(4-2 \beta) \Gamma(6-4 \beta) \Gamma(8-6 \beta)} x^{7-6 \beta}
\end{array}\right)
\end{aligned}
$$

Remark2: Setting $\alpha=\beta=1$ equation (5.11) reduces to classical FPE with solution

$$
u(x, t)=x\left(1+t+\frac{t^{2}}{2 !}+\frac{t^{3}}{3 !}+\ldots\right)
$$

This solution is equivalent to the exact solution in a closed form

$$
u(x, t)=x e^{t}
$$


Table2. The results for exact solution (5.15) and the approximate solution (5.13) of the equation (5.11) obtained using homotopy perturbation natural transform method are shown in the Table for $\alpha=\beta=1$.

\begin{tabular}{|l|l|l|l|}
\hline $\mathrm{T}$ & $\mathrm{X}$ & HPNTM & EXACT \\
\hline 0.2 & 0.25 & 0.3053500 & 0.3053507 \\
\cline { 2 - 4 } & 0.50 & 0.6107000 & 0.6107014 \\
\cline { 2 - 4 } & 0.75 & 0.9160500 & 0.9160521 \\
\cline { 2 - 4 } & 1.00 & 1.2214000 & 1.2214030 \\
\hline 0.4 & 0.25 & 0.3729333 & 0.3729562 \\
\cline { 2 - 4 } & 0.50 & 0.7458667 & 0.7459123 \\
\cline { 2 - 4 } & 0.75 & 1.1188000 & 1.1188690 \\
\cline { 2 - 4 } & 1.00 & 1.4917330 & 1.4918250 \\
\hline 0.6 & 0.25 & 0.4553500 & 0.4555297 \\
\cline { 2 - 4 } & 0.50 & 0.9107000 & 0.9110594 \\
\cline { 2 - 4 } & 0.75 & 1.3660500 & 1.3665890 \\
\cline { 2 - 4 } & 1.00 & 1.8214000 & 1.8221190 \\
\hline
\end{tabular}

The results for exact solutions and approximate solutions for (5.1) and (5.11) obtained by using homotopy perturbation natural transform method, for the special cases $\alpha=1, \beta=1$, are shown in Table 1 and Table2, respectively. It can be seen that the solution obtained by the present method are identical with the exact solution. The accuracy of our approximate solutions can be improved by computing more terms of the approximate solutions. It is to be noted that only the third-order terms of the series of the method are used in evaluating the approximate solutions.

\section{Conclusion}

Within the scope of the homotopy perturbation natural transform method; analytical and approximate solutions of Fokker-Planck equation with space- and time-fractional derivatives are obtained. The numerical result shows that the method used is very simple and straight forward to implement. All the results are calculated by using the symbolic calculus software MATLAB.

\section{References}

[1]. Abbaoui, K., Cherruault,Y., New ideas for providing convergence of decomposition methods, Computers \& mathematics with Applications, 29(7)(1995), 103-108.

[2]. Belgacem, F.B.M., Silambarasan, R., Theory of Natural Transform, Math. Engg. Sci. Aerospace (MESA), 3(1)(2012), 99-124.

[3]. Buet, C., Dellacherie, S., Sentis, R., Numerical solution of an ionic Fokker-Planck equation with electronic temperature, SIAM J. Numer. Anal. 39(2001), 1219-1253.

[4]. Caputo, M., Linear models of dissipation whose Q is almost frequency independent-II, Geophysical Journal International 13(5) (1967), 529-539.

[5]. Chandrasekhar,S., Stochastic problem in physics and astronomy, Review of Modern Physics 15 (1943), 1-89.

[6]. Dubey, R.S., T. Alkahtani, B.S., Atangana, A., Analytical Solution of Space-Time fractional Fokker-Planck Equation by homotopy perturbation Sumudu transform method, Hindawi Publishing corporation, Mathematical Problem in Engineering, Research Article, vol.2015.

[7]. Frank, T.D., Stochastic feedback, nonlinear families of Markov processes, and nonlinear Fokker-Planck equations, Physica A 331(2004), 391-408.

[8]. Fokker, A., The Median energy of rotating electrical dipoles in radiation fields, Annalen Der Physik 43(1914), 810-820.

[9]. Garg, M., Choudhary, S., Solution of space-time fractional Fokker-Planck equation by Homotopy analysis method, Aryabhatta journal of mathematics \& Informatics, 5(2)(2013), 353-364.

[10]. Ghorbani, A., Beyond Adomian polynomials: He's polynomials, Chaos, Solitons and Fractals 39(3)(2009), 1486-1492.

[11]. Harrison, G., Numerical solution of the Fokker-Planck equation using moving finite elements, Numer. Methods Partial Differ. Eqns 4( 1988), 219-232.

[12]. He, J.H., A coupling method of a homotopy technique and a perturbation technique for nonlinear problems, International Journal of Non-linear Mechanics, 35(1)(2000), 37-43.

[13]. He, J.H., Homotopy perturbation technique, Computer Methods in Applied Mechanics and Engineering, 178(3-4)(1999), $257-262$.

[14]. He, J.H., The homotopy perturbation method for nonlinear oscillators with discontinuities, Applied mathematics and computation, $151(1)(2004), 287-292$.

[15]. He, J.H., Wu, X.H., Construction of solitary solution and compacton-like solution by variational iteration method, Chaos Solitons Fractals 29(2006), 108-113.

[16]. Jumarie, G., Fractional Brownian motions via random walk in the complex plane and via fractional derivative, comparison and further results on their Fokker-Planck equations, Choas Solitons fractals 22(2004), 907-925.

[17]. Khan, Zafar H., Khan, waqar A., N- Transform: Properties and Applications, NUST J. Engg. Sci. 1(1) (2008), $127-133$. 
[18]. Kramers, H.A., Brownian motion in a field of force and the diffusion model of chemical reaction, Physica 7(1940), 284-304.

[19]. Liu, F., Anh,V., Turner,I., Numerical simulation for solute transport in fractal porous media, ANZIAM J. (E) 45(2004), 461-473.

[20]. Liu, F., Anh,V., Turner,I., Numerical solution of fractional Fokker-Planck equation, J. Comput. Appl. Math. Volume 166,(2004), 209-219.

[21]. Metzler, R., Barkai, E., Klafter, J., Anomalous diffusion and relaxation close to thermal equilibrium: a fractional Fokker-Planck equation approach, Phys. Rev. Lett. 82(1999), 3563-3567.

[22]. Miller, K.S., Ross, B., An Introduction to the Fractional Calculus Fractional Differential Eqautions. Wiley, New York, 1993.

[23]. Oldham, K.B., Spanier, J., The Fractional Calculus. Academic Press, New York, 1974.

[24]. Risken, H., The Fokker-Planck Equation: Method of Solution and Applications, 1989 (Berlin: Springer)

[25]. Samko, S.G., Kilbas, A.A., Marichev, O.I., Fractional Integrals and Derivatives: Theory and Applications. Gordon and Breach, New York, 1993.

[26]. Silambarasan, R., Belgacem, F.B.M., Applications of Natural transform to Maxwell's equations, Prog. Electromagnetic Research Symposium Proc. Suzhou, China, (2011), 899-902.

[27]. Vanjara, V., Numerical solution of simple Fokker-Planck equation, Appl. Numer. Math. 9(1992), 533-540.

[28]. Xu, Y., Ren, F.Y., Liang, J.R., Qiu, W.Y., Stretched Gaussian symptotic behavior for fractional Fokker-Planck equation on fractal structure in external force fields, Choas Solitons fractals 20(2004), 581-586.

[29]. Yaldirim, A., Analytic approach to Fokker-Planck equation with space- and time- fractional derivatives by means of homotopy perturbation method, Journal of King Saud University (Science) 22(2010), 257-264.

[30]. Zorzano, M.P., Mais, H., Vazquez, L., Numerical solution of two-dimensional Fokker-Planck equations, Appl. Math. Comput. 98(1999), 109-117. 